\title{
On the internal field correction in far-infrared absorption of highly polar molecules in neat liquids and dilute solutions
}

\author{
J. K. Vij \\ Department of Microelectronics \& Electrical Engineering, University of Dublin, Trinity College, Dublin 2, \\ Ireland \\ Yu P. Kalmykov \\ Institute of Radio Engineering and Electronics, Russian Academy of Sciences, Vvedenskii Sq 1, Fryazino, \\ 141120 Russia
}

(Received 17 November 1992; accepted 5 May 1993)

\begin{abstract}
Far-infrared absorption spectra for liquid acetone, methylene chloride, acetonitrile, methyl iodide, and their dilute solutions in cyclohexane at $20^{\circ} \mathrm{C}$ are measured by molecular laser spectrometer. Measurements of dielectric loss of polar liquids and solutions in the frequency range $2-300 \mathrm{GHz}$ are made using a number of different techniques. These two sets of measurements are combined with those made using a Fourier transform spectrometer in order to cover the frequency range up to $250 \mathrm{~cm}^{-1}$ and total integrated absorption intensities are calculated. It is shown that the discrepancy between experimental integrated absorption and the theoretical results given by Gordon's sum rule with the Polo-Wilson internal field factor can be explained in the context of Bossis' theory. This theory gives a better agreement with the experimental integrated absorption intensity for these liquids.
\end{abstract}

\section{INTRODUCTION}

The zero to terahertz frequency spectra of a number of rigid polar molecules in neat liquids and dilute solutions have been studied by a number of investigators (see Refs. 1-6). It is now well established ${ }^{7}$ that the far-infrared absorption of highly polar liquids and solutions is mainly due to the rotational motion of the permanent dipole moment. The integrated dipolar absorption can be expressed in terms of the molecular quantities by Gordon's sum rule. ${ }^{8}$ However, it is also well known that the experimental integrated intensities are usually greater than those calculated using Gordon's sum rule. The discrepancy between the measured and the calculated values cannot be solely accounted by the Polo-Wilson internal field correction factor. ${ }^{9}$ This follows from the fact that Polo-Wilson correction factor is valid only for spherical molecules with an isotropic polarizability. This is practically unrealistic for highly polar molecules. It has been shown by Bossis ${ }^{4}$ that this discrepancy between the theoretical and experimental result may be explained by taking into account both the shape and the anisotropy of the polarizability of the molecule under investigations. Theoretical analysis which generalized the internal field correction of Polo and Wilson, ${ }^{9}$ considerably improves the agreement with experiment.

In the present paper measurements of power absorption coefficient of acetone $\left(\left(\mathrm{CH}_{3}\right)_{2} \mathrm{CO}\right)$ methylene chloride $\left(\mathrm{CH}_{2} \mathrm{Cl}_{2}\right)$, acetonitrile $\left(\mathrm{CH}_{3} \mathrm{CN}\right)$ and methyl iodide $\left(\mathrm{CH}_{3} \mathrm{I}\right)$ in their neat liquid phase and in dilute solutions in cyclohexane are presented in the frequency range $2 \mathrm{GHz}-6$ THz. The measurements have been made using a molecular laser spectrometer and a Fourier transform spectrometer.

The object of this paper is to report high-accuracy farinfrared absorption spectra for four simple polar liquids and their extremely dilute solutions in cyclohexane. This would allow us to systematically test the theory ${ }^{4}$ that provides an explanation for the excess absorption for polar liquids and their dilute solutions, over and above to that predicted by Gordon sum rule with the inclusion of the Polo-Wilson correction factor, in terms of the molecular parameters.

\section{EXPERIMENT}

In the $\mathrm{GHz}$ frequency range ( $2-75 \mathrm{GHz}$ ), methods for measūring $\epsilon^{\prime \prime}(\omega)$ for dilute solutions of polar compounds are based on determining the attenuation suffered by the signal in traversing a given length of the waveguide cell filled with the solution. The microwave techniques and the methods used at these frequencies have been described previously by one of the authors. ${ }^{10}$ Dielectric loss of polar liquids in the frequency range $50-310 \mathrm{GHz}$ (Ref. 11) has been determined using a set of carcinotrons as a source and a bolometer as a detector.

The far-infrared absorption spectra for acetone, methylene chloride, acetonitrile, and methyl iodide in neat liquids and in dilute solutions in cyclohexane $\left(\mathrm{C}_{6} \mathrm{H}_{12}\right)$ at $20^{\circ} \mathrm{C}$ were measured with a molecular laser ${ }^{12}$ system. The dilute solution measurements employed a two-chamber differential celli ${ }^{13}$ whereas a variable thin path length cell was used for neat liquids. The spot wavelengths given in micrometers for which the measurements were made are $1258.3(238.4 \mathrm{GHz}), 447.1(671.0 \mathrm{GHz}), 250.8(1.196$ THz), $191.5(1.567 \mathrm{THz}), 118.8(2.525 \mathrm{THz}), 96.52$ $(3.108 \mathrm{THz})$ and $70.5 \mu \mathrm{m}(4.255 \mathrm{THz})$. These measurements were combined with those made by using a Fourier transform infrared spectrometer (FTS) 113C Bruker spectrometer in the spectral range $80-250 \mathrm{~cm}^{-1}$ and a Grubb Parsons Fourier transform spectrometer.

For each polar liquid, five dilute solutions with varying mole fraction $f_{2}$ in the range 0.2 to $1.5 \%$ were prepared. 
The normalized experimental integrated intensity (IA) $)_{\exp }$ was determined as follows

$$
\text { (IA) })_{\exp }=v_{F}^{-1} \int_{0}^{\text {Band }} \Delta A(\bar{v}) d \bar{v}
$$

where $\bar{v}$ is the wave number in $\mathrm{cm}^{-1}$.

Here $\Delta A(\bar{v})$ is the increment in the absorption coeffcient of the solution over the solvent and $v_{F}$ is the volume fraction of the solute which is given by

$$
v_{F}=\frac{v_{2}}{v_{1}+v_{2}}=\frac{\omega_{2} / \rho_{2}}{\omega_{1} / \rho_{1}+\omega_{2} / \rho_{2}},
$$

where $\omega_{1}$ and $\omega_{2}$ refer to the weights of the solvent and the solute, respectively. Similarly $\rho_{1}$ and $\rho_{2}$ refer to the densities of the solvent and the solute. $v_{F}$ can be calculated directly or related to the mole fraction of the solute as follows. For extremely dilute solutions

$$
\begin{aligned}
v_{F}=\frac{\omega_{2} / \rho_{2}}{\omega_{1} / \rho_{1}} & \cong \frac{\omega_{2}}{\omega_{1}} \frac{\rho_{1}}{\rho_{2}} \\
& =\frac{\omega_{2} / M_{2}}{\omega_{1} / M_{1}} \cdot \frac{M_{2}}{M_{1}} \frac{\rho_{1}}{\rho_{2}} \\
& \cong \frac{\omega_{2} / M_{2}}{\omega_{2} / M_{2}+\omega_{1} / M_{1}} \cdot \frac{M_{2}}{M_{1}} \frac{\rho_{1}}{\rho_{2}} \\
& \cong f_{2} \cdot \frac{M_{2}}{M_{1}} \frac{\rho_{1}}{\rho_{2}},
\end{aligned}
$$

where $f_{2}$ is the mole fraction of the solute, $M_{2}$ and $M_{1}$ are the molecular weights of the solute and the solvent, respectively.

For the liquid under investigations the necessary extrapolation in the high frequency $\left(>250 \mathrm{~cm}^{-1}\right)$ range leads to the error in the integrated intensity of the order $\sim 2 \%-4 \%$. A comparison of our results with those in the literature ${ }^{4,6,14}$ for the common systems shows a good agreement.

Experimental far-infrared spectra of pure liquids and their dilute solutions in cyclohexane are shown in Figs. $1-4$, and their experimental integrated intensities are listed in Table I. Debye time $\left(\tau_{D}\right)$ is defined such that $2 \pi f_{\max } \tau_{D}$ $=1$, where $f_{\max }$ is the frequency for maximum $\epsilon^{\prime \prime}$.

\section{THEORETICAL DESCRIPTION}

The theoretical integrated dipolar absorption IA is usually evaluated as ${ }^{7}$

$$
\mathrm{IA}=\int \alpha(\bar{v}) d \bar{v}=F_{\mathrm{PW}} \mathrm{IA}_{G},
$$

where $\alpha$ is the absorption coefficient,

$$
F_{\mathrm{PW}}=\frac{\left(\epsilon_{\infty}+2\right)^{2}}{3 \sqrt{\epsilon_{\infty}}}
$$

is the Polo-Wilson internal field correction factor, ${ }^{9} \epsilon_{\infty}$ is the value of the dielectric permittivity $\epsilon(\omega)$ at high frequencies,

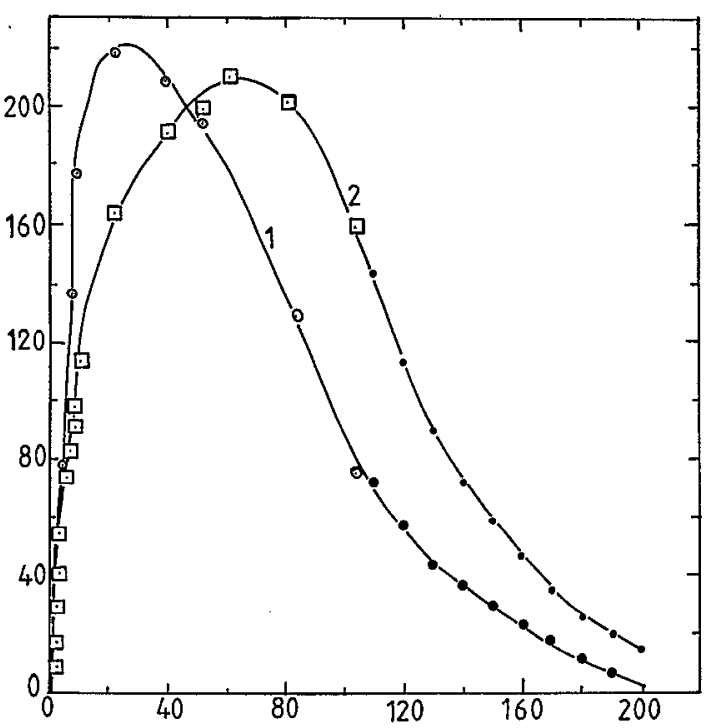

FIG. 1. Plot of power absorption coefficient $A(2 \pi \bar{v} c)$ (neper/cm) vs $\bar{v}\left(\mathrm{~cm}^{-1}\right)$ for acetone at $20 \mathrm{C}$. Curve $1, \Delta A(2 \pi \bar{v} c) / \bar{v}_{F}$ vs $\bar{v}$ for acetone in cyclohexane; $\odot$ laser data, - FTS data; curve 2, $\square$ laser data for pure liquid, - FTS data.

$$
\mathrm{IA}_{G}=\frac{\pi \mu^{2} N}{3 c^{2}}\left(\frac{1}{I_{x}}+\frac{1}{I_{y}}\right)
$$

is given by the Gordon sum rule, ${ }^{8} I_{x}$ and $I_{y}$ are the principal moments of inertia about the $x$ and $y$ axes of the molecule, the dipole moment is assumed to be directed along the $z$ axis, $\mu$ is the permanent dipole moment in units of Debye, $N$ is the molecular number density in $\mathrm{gcm}^{-3}, c$ is the velocity of light.

Equations (3)-(5) do not take into account the shape and the anisotropy of the polarizability of the molecule. An estimation of these factors has been given by Bossis ${ }^{4}$ for the case of the ellipsoidal cavity model. Equation (3) may be written down as follows:

$$
\int \alpha(\bar{v}) d \bar{v}=F_{e}(\mathrm{IA})_{G},
$$

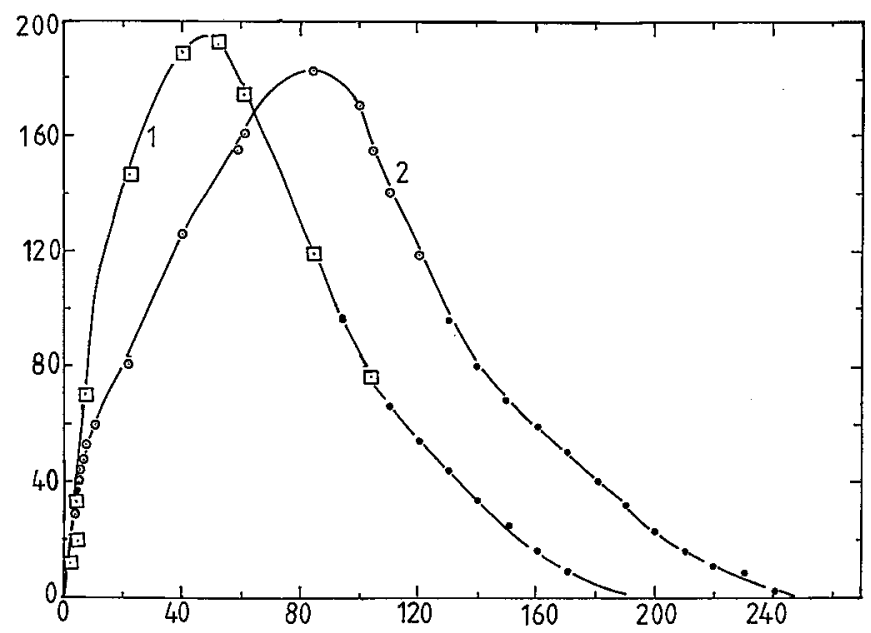

FIG. 2. The same as in Fig. 1 for methylene chloride, curve 1 for dilute solution, $\square$ laser data, - FTS data; curve $2, \odot$ laser data for pure liquid, - FTS data. 


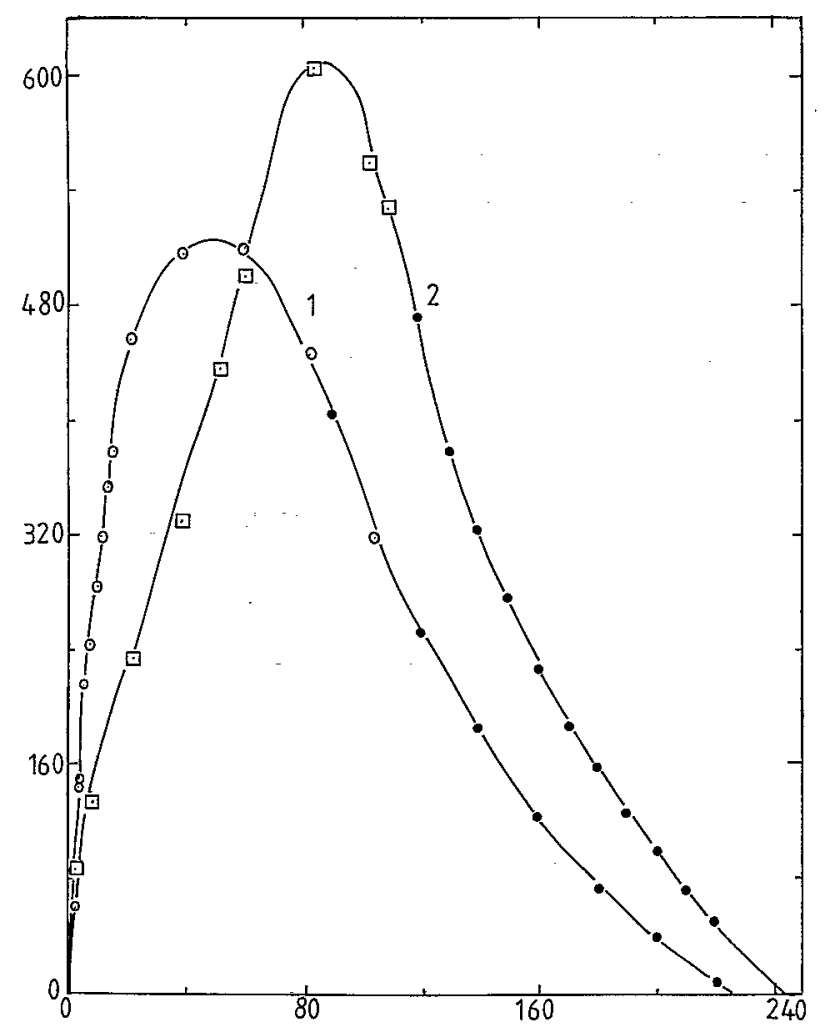

FIG. 3. The same as in Fig. 1 for acetonitrile.

where $F_{e}$ is a correction factor incorporating this anisotropic factor. For dilute solutions of polar liquids in nonpolar solvents, the factor $F_{e}$ is calculated by Bossis. The expression for $F_{e}$ depends on whether $\mu$ lies along or is normal to the symmetry axis of the polarizability.

For the case of pure polar liquids when the dipole moment is considered both along and perpendicular to the revolution axis, the expressions are also derived for $F_{e}$ by Bossis. ${ }^{4}$

It should be noted that formulas ${ }^{4}$ have been derived with the assumption that the shape and the polarizability

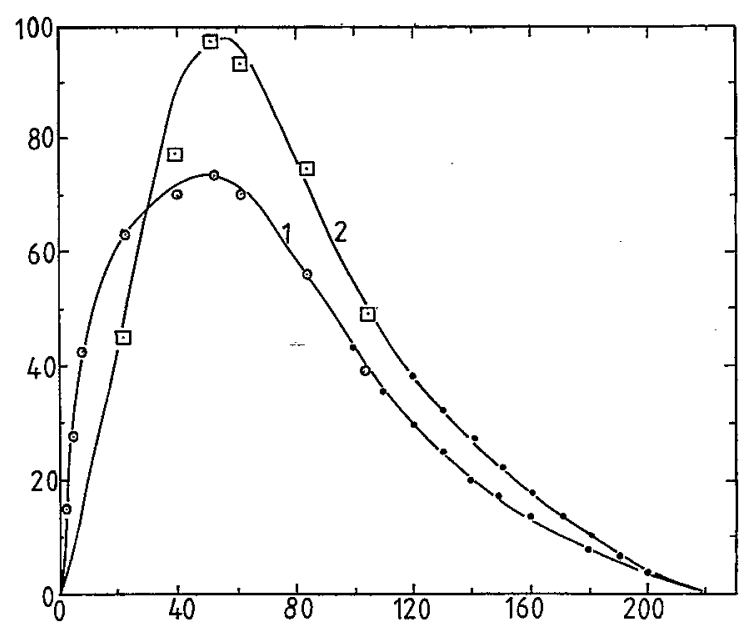

FIG. 4. The same as in Fig. 1 for methyl iodide.
TABLE I. Experimental data for the Debye times $\left(\tau_{\mathrm{D}}\right)$, intgrated absorption (IA) $)_{\exp }$ and $(\text { IA })_{G}$ maximum absorption $\left(\alpha_{\max }\right)$ and corresponding frequencies $\left(\bar{v}_{0}\right)$, and bandwidths $(\Delta \vec{v})$.

\begin{tabular}{|c|c|c|c|c|c|c|}
\hline & $\tau_{\mathbf{D}} / \mathrm{ps}$ & $\begin{array}{l}\text { (IA) } \\
10^{3} \mathrm{~cm}^{-2}\end{array}$ & $\begin{array}{c}(\mathrm{IA})_{G} \\
10^{3} \mathrm{~cm}^{-2}\end{array}$ & $\frac{\alpha_{\max }}{\text { nep. } \mathrm{cm}^{-1}}$ & $\begin{array}{c}\bar{v}_{0} / \\
\mathrm{cm}^{-1}\end{array}$ & $\begin{array}{c}\Delta \bar{v} / \\
\mathrm{cm}^{-1}\end{array}$ \\
\hline \multicolumn{7}{|l|}{ Pure Liquids } \\
\hline Acetone & 3.22 & 24.3 & 12.9 & $208 \pm 4$ & $64 \pm 2$ & 113 \\
\hline $\begin{array}{l}\text { Methylene } \\
\text { chloride }\end{array}$ & 2.07 & 20.5 & 11.7 & $182 \pm 4$ & $87 \pm 2$ & 108 \\
\hline Acetonitrile & 3.62 & 73.4 & 48.6 & $650 \pm 10$ & $90 \pm 2$ & 103 \\
\hline Methyl iodide & 3.62 & 9.2 & 5.5 & $99 \pm 2$ & $57 \pm 2$ & 82 \\
\hline \multicolumn{7}{|c|}{ Solutions in Cyclohexane } \\
\hline Acetone & 1.45 & 20.2 & 12.9 & $220 \pm 4$ & $26 \pm 2$ & 86 \\
\hline $\begin{array}{l}\text { Methylene } \\
\text { chloride }\end{array}$ & 0.57 & 17.1 & 11.7 & $194 \pm 4$ & $48 \pm 2$ & 84 \\
\hline Acetonitrile & 0.64 to 2.39 & 60.7 & 48.6 & $536 \pm 5$ & $47 \pm 2$ & 110 \\
\hline Methyl iodide & 1.84 & 8.5 & 5.5 & $74 \pm 2$ & $51 \pm 2$ & 101 \\
\hline
\end{tabular}

of the molecule can be approximated by two ellipsoids of revolution, which do not exactly coincide for short times and when the permanent dipole moment lies along one of the principal axis. For the permanent dipole moment lying along the axis of greater polarizability, these equations predict an excess absorption which will increase in proportion to the anisotropy in the shape and the polarizability. These equations may be considered as a generalization of the internal field correction factor given by Polo and Wilson (for the case of liquids composed of spherical molecules). One can also use these equations for determining the shape of the cavity that best fits the experimental results. This is useful for determining the volume of the cavity containing the dipolar molecule for the case of dilute solutions.

\section{COMPARISON WITH THE EXPERIMENTAL RESULTS}

From a comparison of the dilute solution and the neat liquid spectra, we find that the frequency of the maximum absorption for dilute solutions decreases compared to that for the neat liquids. This reffects the fact that far-infrared absorption of polar liquids has intermolecular origin. The frequency of maximum power absorption is associated with the mean frequency of molecular librations. ${ }^{6}$ The torsional frequency $\Omega_{0}$ of librations in harmonic approximation is given by Ref. 15:

$$
\Omega_{0}=\left(V_{0} / 4 I_{r}\right)^{1 / 2} \text {, }
$$

where $V_{0}$ is the height of the potential barrier in which a dipole is situated and $I_{r}$ is the reduced moment of inertia of a molecule, $I_{r}=I_{x} I_{y} /\left(I_{x}+I_{y}\right)\left(I_{x}\right.$ and $I_{y}$ are the principal moments of inertia of the molecule and it is assumed that the dipole moment lies along the $z$ axis). An observed increase in the frequency of maximum absorption predicts a considerable increase in the height of the potential barrier $V_{0}$ in going from the dilute solution to the neat liquid (e.g., for acetone the sixfold increase). The dipole-dipole coupling between the molecules in the neat liquids and the intermolecular steric effects seem to be the dominant contributing factors giving rise to an increase in the height of 
the potential barrier. $V_{0}$ is higher in neat liquids due to the larger dipolar torques than for the dilute solutions. The change in the bandwidth of the absorption spectra for the neat liquids from their dilute solutions is reflected in a change of the damping coefficient $\Gamma=$ half-width $/ f_{0}$.

In order to test the theory for the integrated absorption intensity, we compare the experimental internal field correction factor $F_{\text {exp }}$ defined as

$$
F_{\exp }=(\mathrm{IA})_{\mathrm{obs}} /(\mathrm{IA})_{G}
$$

with the theoretical factor $F_{e}$. As in Ref. 4, we have taken molecules with high values of $\mu^{2} / I_{r}$, i.e., light molecules with large dipole moments. This allows us to neglect the absorption from the induced dipole moments. We have chosen acetone, methylene chloride, acetonitrile, and methyl iodide molecules. We have taken acetonitrile for making a comparison with the results in Ref. 4, whereas such detailed and accurate measurements on other systems have not so far, in our view, been reported in the literature before.

Methylene chloride and acetone molecules are asymmetric tops. Nevertheless, we applied the theory ${ }^{4}$ to these molecules assuming that the methylene chloride and acetone molecules can be approximated by prolate and oblate symmetric tops, respectively, with the dipole moment lying perpendicular to the axis of revolution. We have used the same method of the calculation as in Ref. 4.

The mean polarizability $\alpha$ of neat liquids has been calculated from the infrared refractivity using the LorentzLorenz formula:

$$
\alpha=\frac{3 M\left(\epsilon_{\infty}-1\right)}{4 \pi \rho\left(\epsilon_{\infty}+2\right)},
$$

where $M$ is the molecular weight and $\rho$ is the number density.

The anisotropy of polarizability $\beta$ for acetone, methylene chloride, acetonitrile, and methyl iodide has been taken from Ref. 16 where the measurements of $\beta$ in the gas phase have been made. This gives us reliable values of $\beta$ for these systems. For neat liquids we have taken the volume of the cavity $(4 \pi / 3) a_{x} a_{y} a_{z}$ (which enters into the definition of $f_{\gamma}$ ) as equal to the volume per molecule (Onsager's hypothesis); $a_{x}, a_{y}$, and $a_{z}$ are the semiaxes of the ellipsoid. For the case of dilute solutions of polar molecules in cyclohexane, the volume per molecule of the pure dipolar liquid near its melting temperature is taken as the volume of the cavity.

The shape factors $S_{\|}$and $S_{\perp}=\left(1-S_{\|}\right) / 2$ which enter into the calculations depend on the ratio $a_{z} / a_{x}$ as the lengths of the principal axes of the ellipsoid of revolution which are arbitrary. This assumed dependence of the shape factors on the geometry for the case under investigation is an unsatisfactory feature of the analysis. However, values of $S_{\|}$and $S_{\perp}$ may also be estimated from the bond lengths and van der Waal's atomic diameters. As shown in Ref. 4, $F_{e}$ does not strongly depend on the ratio $a_{z} / a_{x}$ because when $a_{z} / a_{x}$ increases, it leads to an increase in $F_{e}$, but also the reaction field factor $f_{\|}$decreases which in turn re-
TABLE II. Molecular weight (M), density $\rho$ and principal moments of inertia $\left(I_{\|}, I_{\perp}\right)$, and dipole moments of organic compounds under investigation.

\begin{tabular}{lclccc}
\hline \hline Material & $\mathrm{M} / \mathrm{g}$ & $\rho^{t} / \mathrm{g} \mathrm{cm}^{3}$ & $\begin{array}{c}I_{\|} \cdot 10^{38} / \\
\mathrm{g} \mathrm{cm}^{2}\end{array}$ & $\begin{array}{c}I_{1} \cdot 10^{38} / \\
\mathrm{g} \mathrm{cm}^{2}\end{array}$ & $\mu / \mathrm{D}$ \\
\hline Acetone & 58.08 & $\begin{array}{l}0.9157^{-94.5} \\
0.7899^{20}\end{array}$ & 1.63 & 0.79 & 2.86 \\
Methylene & 84.93 & $\begin{array}{l}1.539^{-100} \\
\begin{array}{l}1.3266^{20} \\
\text { Chloride }\end{array}\end{array}$ & 2.77 & 0.26 & 1.61 \\
Acetonitrile & 41.05 & $\begin{array}{l}0.8512^{-45} \\
0.8757^{20}\end{array}$ & 0.87 & 0.05 & 3.97 \\
Methyl iodide & 141.94 & $\begin{array}{l}2.530^{-70} \\
2.279^{20}\end{array}$ & 1.10 & 0.05 & 1.64 \\
\hline \hline
\end{tabular}

duces the magnitude of $F_{e}$ because of the anisotropy (if $\beta$ is large enough one can even obtain a decrease in $F_{e}$ when $a_{z} / a_{x}$ increases).

Molecular parameters ${ }^{17,18,20}$ entering into the calculations are summarized in Table II. Comparison of the experimental, Polo-Wilson's and Bossis's internal field correction factors for pure liquids and dilute solutions is given in Table III. This table also lists the principal polarizabilities taken from the literature. ${ }^{16}$

An inspection of Table III reveals that values of $F_{e}$ are closer to the experimental $F_{\text {exp }}$, for all the cases except acetonitrile in cyclohexane. We find a better agreement between the theory and experiment than that already provided by the Polo-Wilson correction factor $F_{\mathrm{PW}}$.

The most sensitive quantity which introduces uncertainty into the determination of $F_{e}$ is the volume of the cavity which arises for the simple reason that the Onsager's hypothesis is only a crude approximation. Accordingly variations in the size and/or of the shape of the cavity is a way of extending the range of the validity of the theory. For example, if we decrease the volume of the cavity of neat acetone by a factor 0.7 , which is in accordance with the calculations of Ref. 19, we find $F_{e}=1.82$, which gives a better agreement with the experimental value $F_{\text {exp }}=1.88$. However, in this case an uncertainty does still exist due to an assumption already made that acetone molecule is approximated by a symmetric top.

TABLE III. Physical parameters of the liquids and a comparison of the

\begin{tabular}{|c|c|c|c|c|c|c|c|c|}
\hline & $S_{\|}$ & $\epsilon_{s}^{20}$ & $\epsilon_{\infty}^{20}$ & $\begin{array}{c}\alpha / 10^{-24} \\
\mathrm{~cm}^{3}\end{array}$ & $\begin{array}{c}\beta / 10^{-24} \\
\mathrm{~cm}^{3}\end{array}$ & $F_{\mathrm{PW}}$ & $F_{e}$ & $F_{\exp }$ \\
\hline \multicolumn{9}{|l|}{ Pure liquids } \\
\hline Acetone & 0.60 & 21.1 & 1.94 & 7.35 & -1.82 & 1.21 & 1.51 & 1.88 \\
\hline $\begin{array}{r}\text { Methylene } \\
\text { chloride }\end{array}$ & 0.20 & 9.03 & 2.13 & 8.17 & 2.65 & 1.27 & 1.37 & 1.75 \\
\hline Acetonitrile & 0.20 & 37.0 & 1.92 & 4.86 & 2.24 & 1.23 & 1.53 & 1.51 \\
\hline Methyl iodide & 0.26 & 6.95 & 2.27 & 7.35 & 2.78 & 1.38 & 1.66 & 1.67 \\
\hline \multicolumn{9}{|c|}{ Dilute solutions in cyclohexane } \\
\hline Acetone & 0.60 & 2.023 & 2.023 & 6.42 & -1.82 & 1.26 & 1.61 & 1.57 \\
\hline $\begin{array}{r}\text { Methylene } \\
\text { chloride }\end{array}$ & 0.20 & 2.023 & 2.023 & 6.53 & 2.65 & 1.26 & 1.38 & 1.46 \\
\hline Acetonitrile & 0.20 & 2.023 & 2.023 & 4.46 & 2.24 & 1.26 & 1.43 & 1.25 \\
\hline Methyl iodide & 0.26 & 2.023 & 2.023 & 7.56 & 2.78 & 1.26 & 1.55 & 1.54 \\
\hline
\end{tabular}
theoretical $\left(F_{\mathrm{PW}}\right.$ and $\left.F_{e}\right)$ and experimental $\left(F_{\text {exp }}\right)$ correction factors. 


\section{SUMMARY}

Precise measurements of the dielectric loss and/or the power absorption coefficient for four highly polar liquids and their dilute solutions in cyclohexane made in the frequency range $2 \mathrm{GHz}-7.5 \mathrm{THz}$ have been reported. A comparsion of the experimental integrated absorption intensities and that calculated using Gordon sum rule with PoloWilson correction factor shows large discrepancies between the results. This discrepancy $(\sim 15 \%$ to $30 \%)$ is explained in terms of the anisotropy of the polarizability, the shape of the dipolar molecule and implicitly on the permittivity of the liquid or dilute solution under investigations.

\section{ACKNOWLEDGMENT}

One of the authors (JKV) is grateful to the late Professor Dr. F. Hufnagel for useful discussions and for allowing access to the experimental facilities.

${ }^{1}$ P. Hindle, S. Walker, and J. Warren, J. Chem. Phys. 62, 3230 (1975). ${ }^{2}$ A. Gershel, I. Dimicoli, J. Joffre, and A. Riou, Mol. Phys. 32, 679 (1976).
${ }^{3}$ C. J. Reid and M. W. Evans, Mol. Phys. 40, 1357 (1980).

${ }^{4}$ G. Bossis, Physica A 110, 408 (1980).

${ }^{5} \mathrm{~J} . \mathrm{K}$. Vij and F. Hufnagel, Chem. Phys. Lett. 150, 211 (1988).

${ }^{6}$ J. K. Vij and F. Hufnagel, J. Phys. Chem. 95, 6142 (1991).

${ }^{7}$ M. W. Evans, G. J. Evans, W. T. Coffey, and P. Grigolini, Molecular Dynamics (Wiley, New York, 1982).

${ }^{8}$ R. G. Gordon, J. Chem. Phys. 38, 1724 (1963).

${ }^{9}$ S. R. Polo and M. K. Wilson, J. Chem. Phys. 23, 2376 (1955).

${ }^{10}$ J. K. Vij and F. Hufnagel, Adv. Chem. Phys. 63, 775 (1985).

${ }^{11}$ J. K. Vij, T. Grochulski, A. Kocot, and F. Hufnagel, Mol. Phys. 72, 353 (1991).

${ }^{12}$ J. K. Vij, F. Hufnagel, M. Helker, and C. J. Reid, IEEE J. Quant. Electron. QE-22, 1123 (1986).

${ }^{13}$ J. K. Vij and F. Hufnagel, J. Phys. E: Sci. Instrum. 22, 749 (1989).

${ }^{14}$ T. Ohbu and S. Ikawa, Mol. Phys. 73, 985 (1991).

${ }^{15}$ W. T. Coffey, P. M. Corcoran, and J. K. Vij, Chem. Phys. Lett. 129, 375 (1986); W. T. Coffey, P. M. Corcoran, and J. K. Vij, Proc. R. Soc. London, Ser. A 410, 61 (1987).

${ }^{16}$ M. P. Boggard, A. D. Buckingham, R. K. Pierens, and A. White, J. Chem. Soc. Faraday Trans. 1 74, 3008 (1978).

${ }^{17} \mathrm{CRCH}$ Handbook of Chemistry and Physics, $62 \mathrm{nd}$ ed. (CRC, Boca Raton, FL).

${ }^{18}$ S. O. Morgan and H. H. Lowry, J. Phys. Chem. 342385 (1930).

${ }^{19}$ V. S. Libov and T. S. Perova, Russ. J. Phys. Chem. 54, 549 (1980).

${ }^{20} \mathrm{~J}$. Timmermans, Physico-Chemical Constants of Pure Organic Compounds (Elsevier, New York, 1950). 